\title{
Needs-Led Research: A Way of Employing User Involvement in Establishing Research Questions on the Trust Model Within Community Home-Based Health Care Services in Norway
}

\section{Ruth-Ellen Slåtsveen ( $\square$ ruthelle@oslomet.no)}

OsloMet HV: OsloMet - storbyuniversitetet Fakultet for helsevitenskap https://orcid.org/0000-00031924-6409

\section{Torunn Wibe}

Centre for Development of Institutional and Home Care Services in the City of Oslo

Liv Halvorsrud

Oslo Metropolitan University: OsloMet - storbyuniversitetet

Anne Lund

Oslo Metropolitan University: OsloMet - storbyuniversitetet

\section{Research article}

Keywords: needs-led research, user involvement, James Lind Alliance, doctoral research, home-based health care services, trust model, trust reform

Posted Date: October 9th, 2020

DOI: https://doi.org/10.21203/rs.3.rs-87428/v1

License: (c) (1) This work is licensed under a Creative Commons Attribution 4.0 International License.

Read Full License 


\section{Abstract}

\section{Background}

This article presents a user involvement process in Norway regarding the performance of the trust model within community home-based health care services. The process, referred to as needs-led research (NLR), is inspired by the James Lind Alliance (JLA) and focuses on bringing together service users, next of kin and clinicians, also referred to as stakeholders, on equal terms to explore research priorities in a prioritysetting partnership.

Method

The following five-step process was followed, in which representatives from service users, next of kin and clinicians were involved in every step. Step 1: A meeting organised in the form of a workshop with service users, next of kin and clinicians to discuss and narrow the scope. Step 2: Steering group meeting. Step 3: Online survey to identify uncertainties regarding the scope. Step 4: Data review and interim prioritisation of research questions. Step 5: Online survey prioritising the final top 10 research questions.

Results

Almost 200 participants contributed during the five steps, 294 submissions were gathered, and 35 participants voted for the top 10 list.

Conclusion

This NLR process was experienced as feasible, and the user involvement shows that it is appropriate and necessary to involve service users, next of kin and clinicians in creating research questions that are relevant to the field.

\section{Plain English Summary}

There is an increased interest in patient and public involvement (PPI) in research aimed at ensuring relevant and needed knowledge. PPI is practised in a variety of ways, and the literature claims that there is no gold standard for how it should be done. This article presents a user involvement process inspired by the James Lind Alliance (JLA) and referred to as a needs-led research (NLR) process. The scope of the process is 'performance of the trust model within community home-based health care services'. The trust model is a new way of organising the home-based health care services. The services are organised in small interdisciplinary teams working with the service users and their next of kin on tailoring services based service users' answers to the question 'What matters to you?'.

Through a five-step process, in which representatives of service users, next of kin and clinicians participate in meetings and online surveys, the goal of the NLR process was to explore research priorities and, ultimately, to develop a final top 10 list of questions relevant to the field of research. This article 
describes the NLR process and discusses the strengths and limitations of the process. We found the process important for identifying relevant research questions regarding a topic on which little research has been done and in which we as researchers have little experience.

\section{Background}

There is evidence of a mismatch between what user groups within the research field find relevant to study and what is actually being done (1-3). Patient and public involvement (PPI) has been proposed as a method to accommodate this (4). PPI in research could be defined as 'research being carried out "with" or "by" members of the public or users rather than "to", "about" or "for" them' (5). Equitable involvement of service users and clinicians in setting research priorities is ethically desirable and is said to improve the quality, relevance and implementation of research (6). There are probably as many approaches to user involvement in research prioritisation as there are priority-setting exercises, and a gold standard is therefore not attainable, nor is it appropriate (7). The James Lind Alliance process focuses on bringing together service users, next of kin and clinicians, also referred to as stakeholders, on equal terms to explore research priorities in a priority-setting partnership (8), and the framework was found fit for the purpose as inspiration $(7,9)$. Although PPI is applied in many studies, detailed descriptions of the process of involving participants/stakeholders are still lacking $(4,10)$. Thus, in this article we describe the process of user involvement with the aim of exploring the research priorities of people with experience of the trust model within community home-based health care services, including health care service users, their next of kin, and clinicians within home-based health care services in a relatively large municipality in Norway (1). The trust model is a new way of organising home-based health care services where trust is described as a strategy and work method aimed at creating: i) services seeking ways of encouraging individual users to become more involved, based on what matters to them, ii) more interaction between the community and the population and between different levels within the community, and iii) greater result orientation and implementation capability (11). This means involving service users and next of kin in decisions that concern them, and trusting clinicians' professional judgement more when it comes to assessing the need for services and for adjusting them if the health of the user changes, making the services individually tailored and more flexible (11). The scope of the study was 'performance of the trust model within community home-based health care services', an area in which limited research has been done in Norway or internationally. No systematic reviews are available, and only a few isolated studies have been conducted. It is a new way of organising the home health care services, and no knowledge exists about what the service providers performing the trust model or the service users or next of kin consider relevant to study, making it appropriate to implement a user involvement process.

\section{Aim}

The aim of this article is to describe a way of doing needs-led research and to assess the value of user involvement. We will also present the top 10 list of research questions the stakeholders found relevant to study regarding 'performance of the trust model within community home-based services'. 


\section{Methods}

Inspired by the James Lind Alliance (JLA) (1), this project's needs-led research (NLR) process is outlined in Figure 1, and consists of five steps: narrowing the theme, steering group meeting, gathering input through a survey, data processing and interim priority setting, and final priority setting. JLA uses the term 'evidence uncertainties' to define the unanswered questions that are identified and prioritised during this process. This article will refer to these as 'input'.

Insert figure 1 here

The scope was 'performance of the trust model within community home-based health care services', with representatives of service users, next of kin and clinicians as stakeholders. Through the five steps, efforts were made to involve an appropriate and balanced representation of stakeholders in order to minimise the risk of overlooking research options and to make sure that the prioritisation corresponds to the needs of those who will benefit from the research priorities (7).

\section{Step 1: Narrowing the theme}

'Performance of the trust model' can be understood in different ways and with variations in content. For the purpose of narrowing the theme, clinicians, service-user and next of kin organisations, members of senior citizens' councils and the Patient Ombudsman were invited to a workshop. The aim was to brainstorm on important aspects regarding the overall theme (7). We wanted the stakeholders to say something about which areas they considered most relevant for research (10). Eighteen representatives participated, evenly distributed among the groups they represented. The first author started the workshop with a presentation of the meeting agenda and some general information including the aim for the workshop. The participants were then divided into three groups. Each group consisted of representatives for service users, next of kin and clinicians, and had a group leader and a minute taker. First, the participants were invited to write down key words on themes, thoughts and experiences regarding the scope. Yellow post-it notes were used for this exercise, which lasted about five minutes. Second, the participants shared and elaborated their notes with the others, and simultaneously systematised the key words they thought belonged together or had similar aspects on a larger white sheet of paper they were given beforehand. Third, they started discussing the notes and were asked to reformulate their grouping into areas or themes using pink post-it notes. The session and discussion in each group lasted for approximately one hour. The aim for this session was to create an opportunity for everyone to talk and elaborate important aspects regarding performance of the trust model. Inspired by qualitative research, the goal was to promote dialogue between the participants (12). They followed each other's stories, offered comments and sometimes different perspectives, which led to full descriptions and perhaps new understandings (12). To ensure a safe environment for all the participants to talk, we started out with small groups. According to Korstjens and Moser (13), the advantage of small groups is that they give participants more time to elaborate on their perspectives and stories, thus they contribute more detailed information. The feedback received afterwards was that the group composition and dynamics were good, and many felt that they benefited from sharing experiences and thoughts with each other in this way. The 
representatives used the opportunity to ask each other questions about the different groups of service users, issues regarding home-based services, and especially the involvement of service users and next of kin within the services. All three groups reported active participation from all the representatives. After a break, everyone gathered and each group presented its topics, thoughts and/or experiences. Many of the areas were similar, and there were no major differences in what the groups talked about. Then there was a discussion in the larger group, and several participants continued to share and problematise the performance of the trust model. This helped create a form of consensus about the areas and themes that had been raised (13). The aim for the meeting was to reach consensus on one or more themes to proceed with in the needs-led research process, but his was not doable. Input in the form of 73 submissions was collected, which provided direction for further focus. In addition, important and useful submissions related to the performance of the trust model were received, and several connections to the field were established.

\section{Step 2: Steering group}

The term steering group is inspired by JLA (1) with the aim of ensuring user involvement in all steps of the process. The group members were not co-researchers, but rather a group that should provide consultation, input, and a connection to the field, as well as influence the steps in the process and the choices made. As experts in their field, they were asked to contribute with their insight and knowledge about the group within the research field they represented. They are familiar with current debates through their affiliation with patient organisations (14) or the home-based services. Two key arguments for involving the research field are to ensure participatory democracy, which is supported by ethical arguments and enshrined in human rights (14). It is also reported to generate better and more relevant decisions, and to create better opportunities and support for further work or changes if needed (14). The steering group consisted of six members: three representatives from the home-based health care services, two service user representatives and one next of kin. Input from the brainstorming session was presented and discussed and served as the basis for the next step. There was consensus amongst the steering group members that the focus should be on the clinician/user level. The steering group members were concerned about whether the trust model provided more accurate and better services, more service user and next of kin involvement, and how the work was performed in practice. This was the starting point for the online survey questions in Step 3. The questions were formulated and accepted by the members present at the steering group meeting. A summary and a draft of the survey were subsequently distributed to all the members, offering them the opportunity to comment on the topics being discussed or on the questions in the survey. We received comments from one member, representing the home-based health care services, related to wording in the survey, and the text was altered accordingly.

\section{Step 3: Gathering input through the online survey}

The survey consisted of some general questions about whether or not the informant was a service user, next of kin or a clinician, age, degree of familiarity with and experience of the trust model. Then followed some information about what the services should contain and what they should deliver within the trust 
model. This was based on the overall standards the municipality itself had published. There were two versions of the main question: one to clinicians and one to service users and next of kin.

The clinicians were asked: 'Home-based health care services should be provided through small teams with more user involvement, based on the question "What matters to you?", with more competent and empowered staff, and with increased interdisciplinary cooperation. If you think about your experience with home-based health care services, what do you think we should investigate within this area?'.

The service users and next of kin were asked: 'Home-based health care services should be provided through small teams where the user and relatives should help determine what the service should be, based on the question "What matters to you?". You should have competent service providers who can make decisions with you when it comes to your services or the services of the person to whom you are next of kin. The staff will work across professions to provide you with good services that are interconnected. If you think about your experience with home-based health care services, what do you think we should investigate within this area?'

The trust model was deliberately not mentioned in the question, based on feedback from the steering group. Most of the service users and next of kin have never heard about the trust model and would therefore most likely lack sufficient knowledge to comment on it. The representatives from the services agreed, since several clinicians wold also likely have difficulties answering what the trust model contained. Therefore, the decision was made to focus on what the services should contain or deliver. The questions were open-ended to encourage full responses regarding experiences and perceptions of service users, next of kin and clinicians (8).

When distributing the online survey, the organisations that participated in the workshop (Step 1) shared information and links to the survey with their members using their existing communication platforms. Centrally located clinicians in the municipality received information and the link via e-mail, and distributed them in appropriate networks within the community. This ensured that all leaders in homebased health care services received the information and were encouraged to both respond to and share the link with their employees.

The questionnaire was published in early January and stayed open for four weeks. 106 respondents and 221 submissions were collected. Most of the respondents came from the home-based health care services (Figure 2).

Insert figure 2 here

Respondents representing clinicians were leaders, occupational therapists, physiotherapists, nurses, nutritionists and unskilled workers.

\section{Step 4: Data processing and interim priority}


The aim for this step was to review the input - 73 submissions from Step 1 and 221 submissions from Step 3 - and organise them into thematic groups. Many of the submissions were formulated more like comments or statements than questions. To accommodate and include these, a thematic approach was found appropriate. The researchers went through all the submissions, grouping and discussing them.

Seven themes were created, and 16 research questions developed during this process (see table 1 for an overview of the themes with examples of input). The steering group was involved in the process of verifying the interpretations and clarifying the questions. They received all the input, groupings, and questions that were created and were asked to give feedback. One single feedback was received, regarding ease of understanding for a couple of the questions, which were altered accordingly.

\section{Table 1. Examples from the thematisation process}




\section{Themes $\quad$ Examples of input}

Interdisciplinary collaboration

- What are success criteria for successful interdisciplinary cooperation?

- How is systematic work in interdisciplinary collaboration facilitated organisationally?

- The importance of interdisciplinarity in the teams?

Competence/ Knowledge

- Has it become easier or more difficult to ensure that personnel with the right competence are assigned to patients where this competence is necessary?

- Core competence: To clarify what competence the service needs for different positions and roles in order to have competent and empowered employees: e.g. observational competence of employees

- Competence to assess the user's functional ability and cognitive status?

Assistive technology and digitalisation in the service
- More on assistive technology because more things are becoming electronic and there are still many who have not understood that it will become more digital with time.

- Training and information about it should be more prioritised.

- Research more on how different departments work on the services
Examples of research questions

How is interdisciplinary collaboration performed in small teams?

What

interdisciplinary competencies do the employees in the teams need, and how do they make best possible use of these competencies?

How are assistive technology and digitalisation implemented and used within interdisciplinary teams?
Organisations and leadership

- How does the trust model contribute to an organisation that provides more tailored and flexible services?

- What significance do organisation and management have for the performance of small interdisciplinary teams?

Early

intervention/

preventive services
- Is there still more focus on nursing than on rehabilitation?

- Do they manage to integrate reablement as well?

- The rehabilitation services in specialist health care are reduced, and it is expected that the municipalities will provide rehabilitation to a greater extent. The districts are falling behind in stepping up rehabilitation services to meet these requirements (of the trust model). How will the districts manage to step up and organise the service to meet users in need of rehabilitation?
How does the trust model ensure early intervention and preventive work?
Exercise of authority and profession
- Roles and responsibilities in the trust model - how has it affected employees and the organisation?

- The term 'empowered employees' and independent professional practice is often used in connection with the
How are roles and responsibilities exercised by employees in the trust model? 
trust model. Do employees feel that they have been given more authority and a greater degree of independence in their professional practice? Do the employees experience wider scope of action?

User and next of kin involvement
- The goal of having small teams and a few people to relate to is not implemented in practice. This causes frustration among both users and next of kin.

- How the services involve relatives in planning, design, implementation and evaluation of the services and how next of kin experience it?

- Whether the trust model gives users an experience of better interdisciplinary collaboration and fewer people to relate to, whether they are assigned a 'primary contact' and whether they have good contact with her/him.
How do users and next of kin experience involvement in the process of allocating services when exercising the trust model?

\section{Step 5: Final priority setting}

The aim for final priority setting was to develop a list of the top 10 research questions on performance of the trust model within community home-based health care services. The aim for the list is to highlight important areas for research, but not necessarily to come up with the specific research questions (1). Invitations were widely distributed to service users, next of kin and clinicians in March, but because of the Covid-19 outbreak the planned workshop for final priority setting had to be cancelled. An online survey was therefore conducted based on the 16 question from the interim priority setting. The respondents were asked to select a maximum of 10 questions they considered most important and were asked to comment on why they considered those questions important. The survey was widely distributed to service users-, and next-of-kin organisations, members of senior citizens' councils, the Patient Ombudsman and clinicians. The survey was open for 14 days. Thirty-five respondents answered, consisting of employees, users, next of kin and others (Figure 3 ).

Insert figure 3 here

'Others' represent e.g. members of senior citizens' councils, chairs of supervisory committees for shortstay institutions and nursing homes, politicians and volunteers. Before the top 10 list was published, it was submitted to the steering group in order to achieve consensus.

\section{Results}

The votes for the top 10 were evenly distributed, all rating above $40 \%$, and the question voted as number two was selected as the main question for this project, i.e.:

How does the trust model contribute to flexible and individually tailored services? 


\section{Discussion}

The aim of this article is to describe a way of performing needs-led research and to assess the value of user involvement. The process was inspired by JLA (1), but due to limited available time and resources, some pragmatic adaptions were made to the framework to make it feasible. Patient involvement is seen to represent a cultural change in healthcare, and requires commitment, time, sensitivity, and a willingness to experiment and learn from mistakes (15). In order to meet some of these challenges, this section will discuss some of the strengths and limitations of the process in order to provide some relevant insight to others who might choose a similar method and to address the knowledge to address the reported knowledge gap regarding challenges and recommendation in PPI processes (16).

We have strived for an appropriate representation of relevant stakeholders during the NLR process to minimise the risk of research questions being overlooked, to foster interest and a wish to be a part of the further project, and to align with the needs of those who will implement and benefit from the research priorities (7). There has been broad participation from the services. Although great efforts were made to include service users with experience and knowledge of the trust model, the number of service user respondents was low, especially when gathering input through the online survey in Step 3. Invitations were distributed online to, and promoted on social media by, interest organisations such as pensioner associations and the Norwegian Public Health Association. In a relatively small-scale project like this, when time and resources are limited, it was not feasible to reach more service users or to run a larger campaign to attract them. Those who participated as service users were representatives, such as senior citizens, members of senior citizens' councils, the ombudsman for senior citizens and members of relevant local government committees for senior citizens. One might ask how they could be said represent the wider user population receiving home-based health care services. However, by comparing their input with that from the next of kin, we see overlapping areas of interest. This may indicate that the user representatives have insight into the services, challenges and knowledge needs of the service users themselves. It is highly likely that we did not reach the weakest voices when conducting this process, i.e. individuals in low socioeconomic groups, with low health literacy or who have no access to the internet and social media (8). Consideration should be given to identifying submissions from the service users separately if conducting a similar process in a larger project.

Critics have argued that, despite its democratic intentions, this way of employing user involvement does not necessarily empower patients, since the researcher retains - and may choose to wield - her power to define what a legitimate research question is and how to answer it (9). This may be a limitation on this process as well. However, attempts have been made to eliminate this by involving the steering group after the interim priority setting, as well after selecting the one top-10 question to be researched, and when the final research questions were developed. Nonetheless, we still cannot claim that the weakest voices have been reached and heard. Another aspect to point out is that the greatest influence during the last steps in the process was held by the authors. To avoid this bias, the steering group members could have actively participated in the process of thematising, interpreting and developing the questions in the interim priority setting, Step 4 . This would raise issues such as increased time consumption, the question of financially 
compensating the members, and finding members with sufficient time and interest to participate in such processes. Time and cost are enlightened in other studies as an important aspect of user involvement in research (17).

An evaluation of the process and of the stakeholders' involvement could have been made to increase the quality and acceptability of the process, and may well have provided insight into the experience of participating in such a process and the perception of its impacts (7).

In order to avoid tokenism and a tick-box approach $(9,18)$ the participants and the feedback given during this process have had a strong influence. Since the researchers have no experience of the trust model or its organisational form, this NLR process ensured that the top 10 question was relevant, and that it was grounded in the day-to-day reality of service users, their next of kin and clinicians $(19,20)$. JLA recommend removing non-questions, such as statements and comments (1), but since many of the submissions received through the questionnaire in Step 3 were not shaped as questions, it was decided to include them. One strength of this process is that every submission could be grouped, but the limitation lay in the interpretation that is needed when grouping them and creating questions that capture the essence. Attempts were made to keep the questions as close to the input as possible, but some adaptions were necessary (see Table 1). All submissions were counted as equally important, regardless of which groups they represented. Input from clinicians was often related to the trust model and its organisation and performance, while input from the service users and next of kin was related to their experiences, but at the same time they wanted more knowledge about performance as well. Although no distinctions were drawn between the user groups when interpreting the input, we found similar interests between them. Consensus was also found in the prioritisation of the top 10 list. Votes for the research question that was finally selected - 'How does the trust model contribute to flexible and individually tailored services?' - received seven votes from service providers, four from next of kin, one from a service user and eight from representatives of users and next of kin (e.g. members of senior citizens' councils, politician, and interest organisations). This distribution of votes underpins the research relevance for the field, and supports Brett et al.'s finding of the positive impact of user involvement on identifying userrelevant topics for research agendas and priorities (17).

Thus, although the outbreak of the Covid-19 pandemic created certain limitations, it may also have created some strengths. JLA recommends that the final priority setting be conducted as a workshop with balanced participation of service users, next of kin and service providers (1), where optional group techniques are presented in order to promote good reflections and discussions. This was not feasible because of the national lockdown during the Covid-19 pandemic, and an online survey was conducted instead. Despite the lack of opportunities for discussion and knowledge sharing within the group, , the online survey attracted more participants, and several of them gave reasons for their chosen prioritisation. At the same time, bias was avoided by preventing the first speaker to set the agenda and influence the opinions of the other participants. The participants could freely vote based on their own opinions, and no-one could know how they voted. The method of prioritising may have contributed to 
providing a safer context for the participants. Fifteen participants had signed up for the planned workshop, whereas 35 participants completed the online survey.

The top 10 research priorities reflect a need to address clinicians' competence and knowledge, their approach to and involvement of service users and next of kin, and the organisational structure of the trust model in research.

In addition to identifying research questions that are relevant to the field of research, user involvement is also seen to fill gaps in researchers' knowledge and to correct assumptions, and thereby avoid bias in researchers' thinking (10). This needs-led research process generated new knowledge for us researchers, not only regarding the trust model and its organisational framework, but also regarding the different perspectives and experiences held by service users, next of kin and clinicians. Without user involvement, the research questions would probably been different.

\section{Conclusion}

In a time when PPI is a goal in every study, this paper provides an example of how to create research questions that are relevant for clinicians, service users and next of kin, and for the service providers themselves regarding 'performance of the trust model within community home-based health care services'. This article shows that it is feasible to use JLA as a framework for developing research questions in small-scale studies. It is our hope that the top 10 list will lead to future research that will address issues of importance for performing the trust model, which is a relative new way of organising community health care services in Norway.

\section{Abbreviations}

PPI: Patient and public involvement

JLA: James Lind Alliance

NLR: Needs-led research

\section{Declarations}

\section{Acknowledgements}

We would like to thank everyone who participated in our needs-led research process for contributing their knowledge and experiences.

\section{Authors' contributions}

RES has made substantial contributions to the conception and design of the work and led the needs-led research and writing process. RES has also contributed to acquisition and interpretation of data and has 
approved the submitted version and has agreed on to be personally accountable for own contributions and ensured that questions related to the accuracy are appropriately investigated, resolved and documented in the literature.

TW has made substantial contributions to the design of the work, acquisition and interpretation of data and has approved the submitted version and has agreed on to be personally accountable for own contributions and ensured that questions related to the accuracy are appropriately investigated, resolved and documented in the literature.

LH has made substantial contributions to the design of the work, acquisition and interpretation of data and has approved the submitted version and has agreed on to be personally accountable for own contributions and ensured that questions related to the accuracy are appropriately investigated, resolved and documented in the literature.
AL has made substantial contributions to the conception and design of the work, acquisition and interpretation of data and has approved the submitted version and has agreed on to be personally accountable for own contributions and ensured that questions related to the accuracy are appropriately investigated, resolved and documented in the literature.

\section{Ethics approval and consent to participate}

According to Norwegian law, approval from the Norwegian National Research Ethics Committees was not required, but the study was registered with the Norwegian Centre for Research Data (NSD), project number 382861.

\section{Consent for publication}

The manuscript does not contain any individual person's data in any form.

\section{Competing interests}

The authors declare that they have no competing interests.

\section{Availability of data}

The material in this project is material from workshops and surveys, all in Norwegian.

\section{Funding}

No funding was provided for this work.

\section{Author details}

RES occupational therapist and a PhD student at Department of Occupational Therapy, Prosthetics and Orthotics, Faculty of Health Science at OsloMet - Oslo Metropolitan University. 
AL Professor in occupational therapy at Department of Occupational Therapy, Prosthetics and Orthotics, Faculty of Health Science at OsloMet - Oslo Metropolitan University.

LH Professor, Cand San, RN, OsloMet - Oslo Metropolitan University, Faculty of Health Science, Department of Nursing and Health Promotion

TW PhD, MSc, RN, currently working as consultant at the Centre for Development of Institutional and Home Care Services in the City of Oslo.

\section{References}

1. Research NIfH. The James Lind Alliance Guidebook Version 9. 2020.

2. Glasziou P, Chalmers I. Research waste is still a scandal-an essay by Paul Glasziou and lain Chalmers. BMJ. 2018;363:k4645.

3. Crowe S, Fenton M, Hall M, Cowan K, Chalmers I. Patients', clinicians' and the research communities' priorities for treatment research: there is an important mismatch. Research involvement and engagement. 2015;1(1):2.

4. Dawson S, Ruddock A, Parmar V, Morris R, Cheraghi-Sohi S, Giles S, et al. Patient and public involvement in doctoral research: reflections and experiences of the PPI contributors and researcher. Research involvement and engagement. 2020;6(1):1-13.

5. research NIfh. What is public involvement in research? NHIR/INVOLVE2020 [updated 2020.07.09; cited 2020 2020.07.09]. Available from: https://www.invo.org.uk/find-out-more/\%20what-is-publicinvolvement-in-research-2/.

6. Alex P, Bridget St G, Mark F, Sally C, Lester F. Development of a new model to engage patients and clinicians in setting research priorities. Journal of health services research \& policy. 2014;19(1):12-8.

7. Viergever R, Olifson S, Ghaffar A, Terry R. A checklist for health research priority setting: nine common themes of good practice. Health Research Policy And Systems. 2010;8(1):36.

8. Nygaard A, Halvorsrud L, Linnerud S, Grov EK, Bergland A. The James Lind Alliance process approach: scoping review. BMJ Open. 2019;9(8):e027473.

9. Greenhalgh T, Hinton L, Finlay T, Macfarlane A, Fahy N, Clyde B, et al. Frameworks for supporting patient and public involvement in research: Systematic review and co-design pilot. Health Expectations. 2019;22(4):785-801.

10. Staley K, Barron D. Learning as an outcome of involvement in research: what are the implications for practice, reporting and evaluation? Res Involv Engagem. 2019;5(1):14-9.

11. Eide T, Gullslett MK, Nilsen E, Dugstad JH, Eide H. Tillitsmodellen : hovedpilotering i Oslo kommune 2017-18. Universitetet i Sørøst-Norge; 2018.

12. Tjora AH. Kvalitative forskningsmetoder i praksis. 3. utg. ed. Oslo: Gyldendal akademisk; 2017.

13. Korstjens I, Moser A. Series: Practical guidance to qualitative research. Part 2: Context, research questions and designs. Eur J Gen Pract. 2017;23(1):274-9. 
14. Uhm S, Liabo K, Stewart R, Rees R, Oliver S. Patient and public perspectives shaping scientific and medical research: panels for data, discussions, and decisions. Patient Intelligence. 2012;4:1-.

15. Pepper L. Patient and public involvement in sexual and reproductive health: a new editor, and a new tool. BMJ Sexual \& Reproductive Health. 2018;44(4):237-8.

16. Pii KH, Schou LH, Piil K, Jarden M. Current trends in patient and public involvement in cancer research: A systematic review. Health Expect. 2019;22(1):3-20.

17. Brett J, Staniszewska S, Mockford C, Herron-Marx S, Hughes J, Tysall C, et al. A Systematic Review of the Impact of Patient and Public Involvement on Service Users, Researchers and Communities. Patient. 2014;7(4):387-95.

18. Malterud K, Elvbakken KT. Patients participating as co-researchers in health research: A systematic review of outcomes and experiences. Scandinavian journal of public health. 2019:140349481986351.

19. Snow R, Crocker JC, Crowe S. Missed opportunities for impact in patient and carer involvement: a mixed methods case study of research priority setting. Research Involvement and Engagement. 2015;1(1):7.

20. Staley K. 'Is it worth doing?' Measuring the impact of patient and public involvement in research. Res Involv Engagem. 2015;1(1):6.

\section{Figures}

\section{Needs-led research process 'performance of the trust model within home-based health care services'}

\section{Narrowing the \\ theme \\ Workshop; 18 \\ participants, 73 \\ submissions}

\section{Steering group meeting \\ 2 user, 1 next of - \\ kin, 3 service providers. \\ Consensus about the questionnaire}

Gathering input through survey

106 respondents, 221 submissions (294 submissions when including those from Step

1)

\section{Data processing and interim priority setting \\ Thematic aproach; 7 themes with together 16 research questions}

Final priority setting

Online survey; 35 respondents, top 10 list created

\section{Figure 1}

NLR process flowchart 


\section{RESPONDENTS}

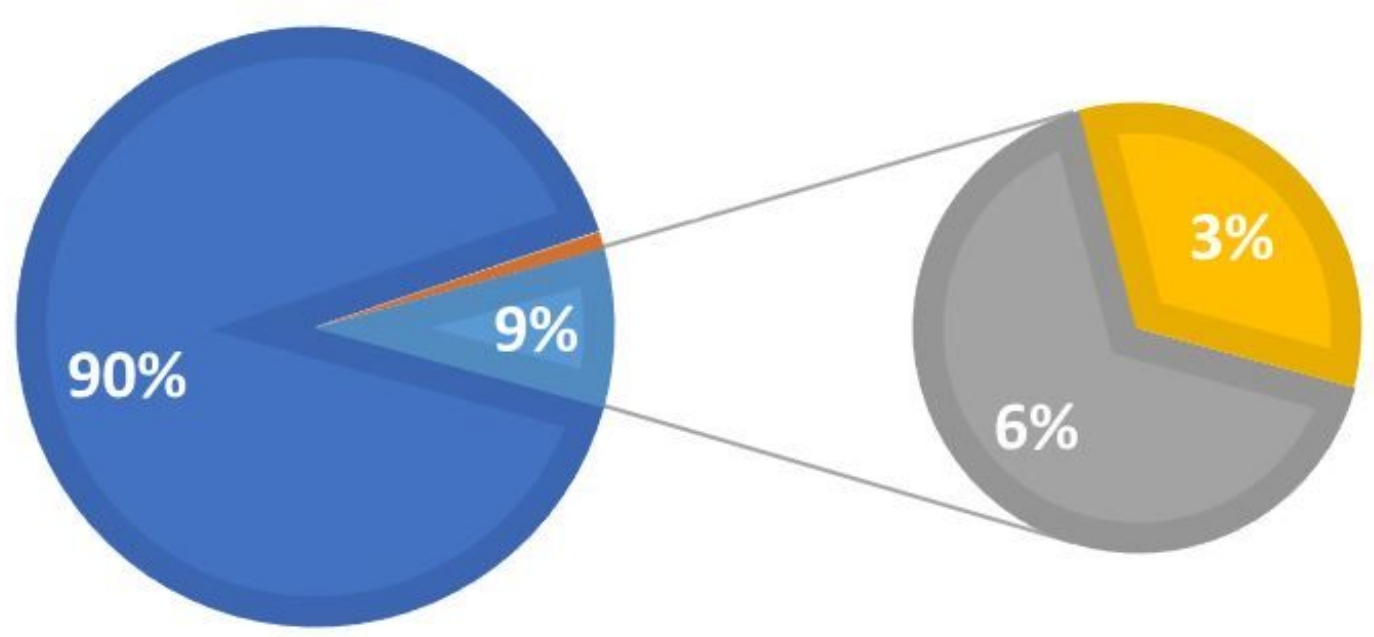

Clinicians $\quad$ Users $\quad$ Next of kin $\quad$ Others

Figure 2

Distribution of respondents 


\section{Respondents}

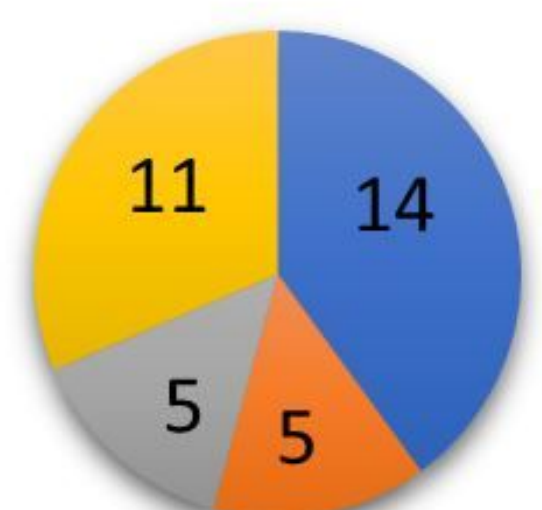

\section{- Clinicians Users \\ Next of kin $\square$ Others}

Figure 3

Distribution of respondents in final priority setting. 
Final top 10: Performance of the trust model

How do service users experience the services after implementation of the trust model?

How does the trust model contribute to flexible and individually tailored services?

How does the trust model, and work in small teams contribute to an experience of personal continuity for users and clinicians?

How do users and next of kin experience involvement related to allocation of services in the context of the trust model?

How does the communication between user, next of kin and clinicians take

place in the performance of the trust model?
What interdisciplinary competence is necessary to possess and how do the teams make the best possible use of this competence?

What significance does organization and management have for the practice of small interdisciplinary teams?

How do clinicians facilitate user involvement in performing the trust model?

How are roles and responsibilities exercised by clinicians in the trust model?

How does the trust model ensure early intervention and prevention?

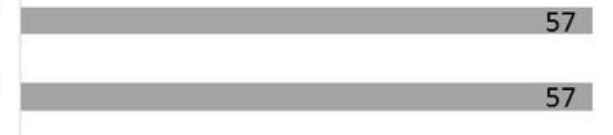

\section{Figure 4}

The top 10 research question regarding performance of the trust model, \% voting. 